\title{
SOLUCIÓN NUMÉRICA PARA UNA ECUACIÓN DIFERENCIAL PARCIAL HIPERBÓLICA: LA ECUACIÓN LA ONDA
}

\author{
NUMERICAL SOLUTION FOR A DIFERENCDIAL PARTIAL HYPERBOLIC \\ EQUATION: THE EQUATION THE WAVE
}

\author{
Luis Andrés Amaya Cedrón ${ }^{13}$
}

\section{RESUMEN}

En la investigación, la solución numérica para una ecuación diferencial parcial hiperbólica, como es la ecuación la onda, es muy aplicable a diversos casos de la Física e Ingeniería. Se estudiará primero la definición de una ecuación diferencial Parcial hiperbólica. Luego estudiaremos la ecuación de la onda, sus condiciones de frontera y su dominio, para finalmente realizar la solución numérica de esta ecuación, la cual será implementada mediante el software de cálculo simbólico Matlab.

Concluimos que es importante él método de solución numérica, pues este método es utilizado en las carreras profesionales.

Palabras claves: Ecuación diferencial parcial, ecuación de la onda, condiciones de frontera.

\section{ABSTRACT}

In research, the numerical solution for a hyperbolic partial differential equation, such as the wave equation, is very applicable to several cases of Physics and. The definition of a hyperbolic partial differential equation will be studied first. Then we will study the equation of the wave, its boundary conditions and its domain, to finally make the numerical solution of this equation, which will be implemented through the Matlab symbolic calculation software (matrix laboratory: symbolic calculation software).

We conclude that the numerical solution method is important, because this method is used in professional careers

Keywords: Partial differential equation, wave equation, boundary conditions.

\footnotetext{
${ }^{13}$ Docente de la UNJBG - Tacna
} 


\section{INTRODUCCIÓN}

Ecuaciones diferenciales parciales

Una ecuación diferencial parcial (EDP) es aquella ecuación diferencial en donde la variable dependiente (función desconocida) deberá ser una función de por lo menos dos variables independientes ya que de no ser así no aparecerían derivadas parciales. La importancia de este tema es que prácticamente en todos los fenómenos que se estudian en ingeniería y en otras ciencias, aparecen más de dos variables y su modelación matemática conduce frecuentemente a una EDP. El modelo general de una EDP a estudiar es:

$A(x, y) \frac{\partial^{2} f}{\partial x^{2}}+B(x, y) \frac{\partial^{2} f}{\partial x \partial y}+C(x, y) \frac{\partial^{2} f}{\partial y^{2}}=F\left(x, y, f, \frac{\partial f}{\partial x}, \frac{\partial f}{\partial y}\right)$

donde $A, B$ y $C$ son funciones de dos variables que serán consideradas constantes, se llama casi-lineal; hay tres tipos de ecuaciones casi-lineales.:

Si $B^{2}-4 A C<0$, la EDP se llama elíptica.

Si $B^{2}-4 A C=0$, la EDP se llama parabólica.

Si $B^{2}-4 A C>0$, la EDP se llama hiperbólica (Hurtado y Domínguez, 1997, p.527).

Como ejemplo de una ecuación elíptica tenemos a la ecuación de Laplace.

$\frac{\partial^{2} f}{\partial x^{2}}+\frac{\partial^{2} f}{\partial y^{2}}=0 ; \quad \mathrm{A}=1 ; \mathrm{B}=0 ; \quad \mathrm{C}=1$; luego

$\mathrm{B}^{2}-4 \mathrm{AC}=0^{2}-4(1)(1)=-4<0$,

(Hurtado y Domínguez, 1997, p.527).

Como ejemplo de una ecuación parabólica tenemos a la ecuación del calor.

$$
\frac{\partial T}{\partial t}=\alpha \frac{\partial^{2} T}{\partial x^{2}} ; \quad \mathrm{A}=1 ; \quad \mathrm{B}=0 ; \quad \mathrm{C}=0 ; \quad \text { luego }
$$

$\mathrm{B}^{2}-4 \mathrm{AC}=0^{2}-4(1)(0)=0, \quad$ (Burden $\mathrm{y}$ Faires, 2007, p.702) y (Hurtado y Domínguez, 1997, p.528).

Como ejemplo de una ecuación hiperbólica tenemos la ecuación de onda.

$\frac{\partial^{2} f}{\partial x^{2}}=\frac{1}{c^{2}} \frac{\partial^{2} f}{\partial t^{2}} ; \quad \mathrm{A}=1 ; \quad \mathrm{B}=0 ; \quad \mathrm{C}=-1 / \mathrm{c}^{2} ;$ luego

$\mathrm{B}^{2}-4 \mathrm{AC}=0^{2}-4(1)\left(-1 / \mathrm{c}^{2}\right)=\frac{4}{\mathrm{c}^{2}}>0$,

(Chapra, 2007, p.860).

Según Chapra (20017, p.859), El Orden de una EDP es el de la derivada parcial de mayor orden que aparece en la ecuación. Por ejemplo:

$\frac{\partial^{2} u}{\partial x^{2}}+2 x y \frac{\partial^{2} u}{\partial y^{2}}+u=1$; es de segundo orden $\mathrm{y} u=\mathrm{u}(\mathrm{x}, \mathrm{y})$

$\frac{\partial^{3} u}{\partial x^{2} \partial y}+x \frac{\partial^{2} u}{\partial y^{2}}+8 u=5 y$; es de tercer orden

\section{Aproximaciones de las ecuaciones diferenciales parciales por diferencias finitas.}

Las aproximaciones de las derivadas mediante diferencias finitas no son restringidas a funciones de una sola variable, cuando se tiene una función de dos variables (Ejemplo: $\mathrm{T}(\mathrm{x}, \mathrm{t})$ ) también se pueden aproximar mediante diferencias finitas:

Según Hurtado y Dominguez (2005, p.533) Aproximación con diferencias centrales para segunda derivada 


$$
\begin{aligned}
& \left.\frac{\partial^{2} T}{\partial x^{2}}\right|_{\left(x_{i}, t_{j}\right)} \approx \frac{T\left(x_{i}+a, t_{j}\right)-2 T\left(x_{i}, t_{j}\right)+T\left(x_{i}-a, t_{j}\right)}{a^{2}} \\
& \left.\frac{\partial^{2} T}{\partial t^{2}}\right|_{\left(x_{i}, t_{j}\right)} \approx \frac{T\left(x_{i}, t_{j}+b\right)-2 T\left(x_{i}, t_{j}\right)+T\left(x_{i}, t_{j}-b\right)}{b^{2}} \\
& \left.\frac{\partial^{2} T}{\partial x \partial t}\right|_{\left(x_{i}, t_{j}\right)} \approx \frac{T\left(x_{i}+a, t_{j}+b\right)-T\left(x_{i}-a, t_{j}+b\right)+T\left(x_{i}+a, t_{j}-b\right)}{4 a b}
\end{aligned}
$$

\section{MATERIALES}

En este trabajo se utilizaron materiales bibliográficos acerca de Ecuaciones diferenciales parciales, también se trabajó con material bibliográfico sobre el software de cálculo simbólico Matlab

\section{MÉTODO}

Se realizará el estudio bibliográfico acerca de Ecuaciones diferenciales parciales y sus aplicaciones a la Física e Ingeniería. Las aplicaciones a la Ecuación de la onda serán procesadas con el software de cálculo simbólico Matlab.

\section{ECUACIÓN DE LA ONDA}

Según Burden y Faires (2007, p.693). La EDP como problema de valores en la frontera (Ecuación de la Onda), está dada por:

$$
\begin{array}{lll}
\frac{\partial^{2} U}{\partial t^{2}}(x, t)-v^{2} \frac{\partial^{2} U}{\partial x^{2}}(x, t)=0, & 0<\mathrm{x}<L ; & 0<\mathrm{t} \\
\frac{1}{v^{2}} \frac{\partial^{2} U}{\partial t^{2}}(x, t)=\frac{\partial^{2} U}{\partial x^{2}}(x, t)=0, & 0<\mathrm{x}<L ; & 0<\mathrm{t}
\end{array}
$$

Sujeto a condiciones:

$\mathrm{U}(0, \mathrm{t})=\mathrm{U}(\mathrm{L}, \mathrm{t})=0 ; \quad 0<\mathrm{t}$

$$
\mathrm{U}(\mathrm{x}, 0)=\mathrm{f}(\mathrm{x}) \quad \text { y } \quad \frac{\partial U}{\partial x}(x, 0) \quad 0 \leq \mathrm{x} \leq L
$$

La cual es una ecuación hiperbólica; puesto que $A=1 ; \quad B=0 ; \quad C=-1 / v^{2}$; (hagamos $v=c$ ), luego:

$$
\mathrm{B}^{2}-4 \mathrm{AC}=0^{2}-4(1)\left(-1 / \mathrm{c}^{2}\right)=\frac{4}{\mathrm{c}^{2}}>0
$$

La ecuación quedaría:

$$
\frac{\partial^{2} U}{\partial t^{2}}(x, t)-c^{2} \frac{\partial^{2} U}{\partial x^{2}}(x, t)=0, \quad 0<\mathrm{x}<L ; \quad 0<\mathrm{t}
$$

Esta ecuación es la forma general de la ecuación que describe a las ondas en dimensión 1+1: "La segunda derivada del desplazamiento de onda $U$ respecto a la coordenada $x$ es igual al inverso de la velocidad de propagación de la onda al cuadrado $\left(\frac{1}{c^{2}}=\frac{1}{v^{2}}\right)$ por la segunda derivada del desplazamiento de esta con respecto al tiempo." (Burden y Faires, 2007)

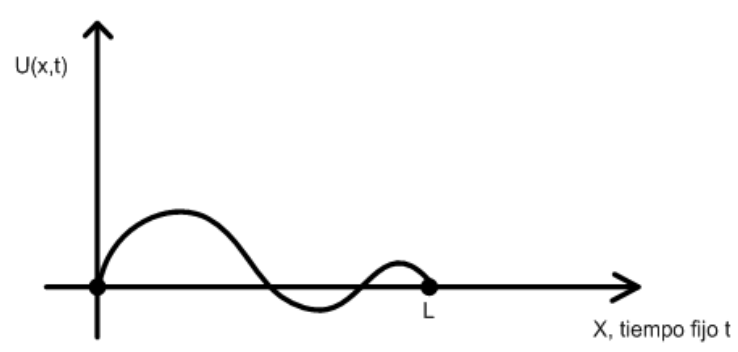

Figura 1. Ecuación de la onda unidimensional

Fuente: (Burden y Faires, 2007, p.693)

Se cumple queU $\left(\left(x_{i}, t_{j}\right)\right)=U\left(x_{i}, t_{j}\right)=U_{i, j}$ y el dominio, los puntos: $\left(x_{i}, t_{j}\right)$.

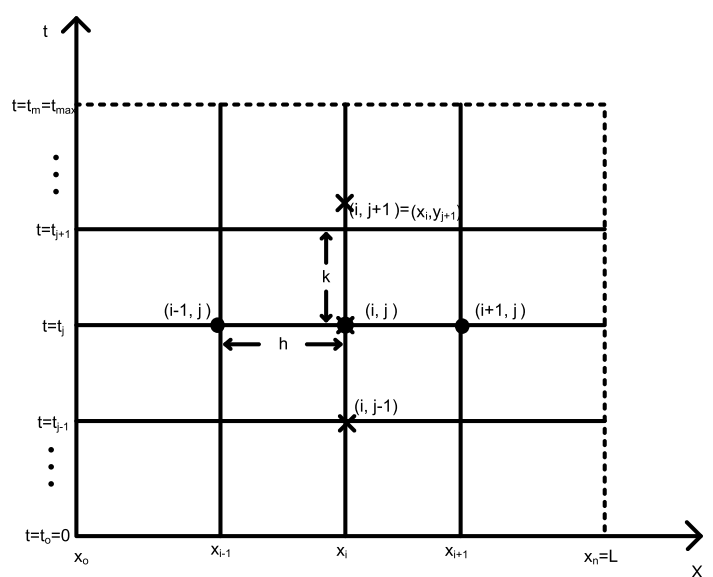

Figura 2. Nodos usados de la malla Fuente: (Hurtado, 1997)

$$
\begin{array}{ll}
i=0,1,2,3, \ldots, n . & x_{i}=i h \\
j=0,1,2,3, \ldots & t_{j}=j k
\end{array}
$$


$\left.\frac{\partial^{2} U}{\partial x^{2}}\right|_{\left(x_{i}, t_{j}\right)} \approx \frac{U\left(x_{i}+h, t_{j}\right)-2 U\left(x_{i}, t_{j}\right)+U\left(x_{i}-h, t_{j}\right)}{h^{2}}$

$\left.\frac{\partial^{2} U}{\partial t^{2}}\right|_{\left(x_{i}, t_{j}\right)} \approx \frac{U\left(x_{i}, t_{j}+k\right)-2 U\left(x_{i}, t_{j}\right)+U\left(x_{i}, t_{j}-k\right)}{k^{2}}$

Ahora se sustituye estas ecuaciones en $\frac{\partial^{2} U}{\partial t^{2}}(x, t)-c^{2} \frac{\partial^{2} U}{\partial x^{2}}(x, t)=0$, válida en todo el dominio de definición resultando:

$\frac{U_{i, j+1}-2 U_{i, j}+U_{i, j-1}}{k^{2}}-c^{2} \cdot \frac{U_{i+1, j}-2 U_{i, j}+U_{i-1, j}}{h^{2}}=0$

Haciendo $\quad \lambda=\frac{c k}{h} \Rightarrow \lambda^{2}=\left(\frac{c k}{h}\right)^{2}$;

podemos escribir la Ecuación como:

$U_{i, j+1}-2 U_{i, j}+U_{i, j-1}-\lambda^{2} \cdot U_{i+1, j}+2 \lambda^{2} U_{i, j}-\lambda^{2} U_{i-1, j}=0$

Para $i=1,2,3, \ldots, n-1 ; j=1,2, \ldots$

Con condiciones de frontera:

$$
\left\{\begin{array}{l}
\mathrm{U}\left(0, \mathrm{t}_{\mathrm{j}}\right)=\mathrm{U}_{0, \mathrm{j}}=0 \\
\mathrm{U}\left(\mathrm{L}, \mathrm{t}_{\mathrm{j}}\right)=\mathrm{U}_{\mathrm{n}, \mathrm{j}}=0 \\
\mathrm{x}_{0}=0 ; \mathrm{x}_{\mathrm{n}}=L
\end{array}\right\} \text { para } \mathrm{j}=1,2, \ldots
$$

La condición Inicial: $U\left(x_{i}, 0\right)=f(x)$; para $i=1,2,3, \ldots \quad t_{0}=0$

Según Hurtado (1997), se escribe en forma Matricial

$$
\begin{aligned}
{\left[\begin{array}{c}
U_{1, j+1} \\
U_{2, j+1} \\
\vdots \\
\vdots \\
U_{n-1, j+1}
\end{array}\right] } & {\left[\begin{array}{ccccc}
2\left(1-\lambda^{2}\right) & \lambda^{2} & 0 & \cdots & 0 \\
\lambda^{2} & 2\left(1-\lambda^{2}\right) & \lambda^{2} & \ddots & \vdots \\
0 & \ddots & \ddots & \ddots & 0 \\
\vdots & \ddots & \ddots & \ddots & \lambda^{2} \\
0 & \cdots & 0 & \lambda^{2} & 2\left(1-\lambda^{2}\right)
\end{array}\right]\left[\begin{array}{c}
U_{1, j} \\
U_{2, j} \\
\vdots \\
\vdots \\
U_{n-1, j}
\end{array}\right] } \\
& -\left[\begin{array}{c}
U_{1, j-1} \\
U_{2, j-1} \\
\vdots \\
\vdots \\
U_{n-1, j-1}
\end{array}\right]
\end{aligned}
$$

Como el (j+1)-ésimo paso necesita jésimo y (j-1)-ésimo pasos.

Es decir para el paso $(j+1)=2$, necesita de $\mathrm{j}=0$ y $\mathrm{j}=1$;

a) Para $j=0$ esta dado por $U\left(x_{i}\right.$, $0)=f\left(x_{i}\right)$.

b) Pero para $j=1$ se necesita la condición inicial:

$$
\frac{\partial U}{\partial t}(x, 0)=g(x) \quad 0 \leq x \leq L
$$

Se puede reemplazar por:

$$
\begin{aligned}
& \frac{\partial U}{\partial t}(x, 0) \approx \frac{U\left(x_{i}, t_{1}\right)-U\left(x_{i}, 0\right)}{k} \\
& \frac{\partial U}{\partial t}(x, 0) \approx \frac{U_{i, 1}-U_{i, 0}}{k} \Rightarrow \\
& U_{i, 1} \approx U_{i, 0}+k \frac{\partial U}{\partial t} \Rightarrow \\
& U_{i, 1} \approx U_{i, 0}+k g\left(x_{i}\right)
\end{aligned}
$$

Para $i=1,2,3, \ldots$ con error $O(k)$.

Si quisiéramos una mejor aproximación con error $\mathrm{O}\left(\mathrm{k}^{3}\right)$, lo reemplazaríamos por:

$$
\begin{aligned}
U_{i, 1} \approx & \left(1-\lambda^{2}\right) f\left(x_{i}\right)+\frac{\lambda^{2}}{2} f\left(x_{i+1}\right) \\
& +\frac{\lambda^{2}}{2} f\left(x_{i-1}\right)+k g\left(x_{i}\right)
\end{aligned}
$$

Aplicación: Para el problema

$\frac{\partial^{2} \mathrm{U}}{\partial \mathrm{t}^{2}}(\mathrm{x}, \mathrm{t})-4 \frac{\partial^{2} \mathrm{U}}{\partial \mathrm{x}^{2}}(\mathrm{x}, \mathrm{t})=0, \quad 0<\mathrm{x}<1, \quad 0<\mathrm{t}$

Con las condiciones de frontera:

$$
\mathrm{U}(0, \mathrm{t})=\mathrm{U}(1, \mathrm{t})=0, \quad \text { para } 0<\mathrm{t}
$$

Con las condiciones iníciales:

$\mathrm{U}(\mathrm{x}, 0)=\operatorname{sen}(\pi \mathrm{x}) \quad 0 \leq \mathrm{x} \leq 1, \quad$ y $\quad \frac{\partial \mathrm{U}}{\partial \mathrm{t}}(\mathrm{x}, 0)=0, \quad 0 \leq \mathrm{x} \leq 1$

Con $n=10$ (para el programa $n=11$ columnas), entonces $h=(1-0) /(11-$ $1)=0.1, y \mathrm{~m}=20$ (para el programa $\mathrm{m}=21$ filas), $k=(1-0) /(21-1)=0.05$; alfa $=2$.

\section{Dominio de $U\left(x_{i}, t_{j}\right)$}

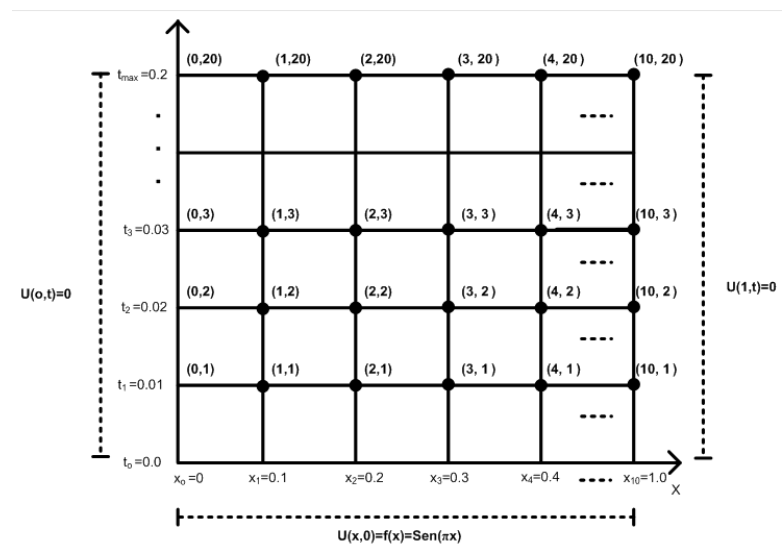

Figura 3: Nodos en el Dominio Fuente: Propia 
Condiciones:

1. Las condiciones de frontera $\mathrm{U}(0, \mathrm{t})=\mathrm{U}(1, \mathrm{t})=0, \quad$ para $0<\mathrm{t}$

Nos proporcionan la información que en los extremos del objeto a desplazarse siempre estará fijo.

2. La condición Inicial:

$\mathrm{U}(\mathrm{x}, 0)=\operatorname{sen}(\pi \mathrm{x}) \quad 0 \leq \mathrm{x} \leq 1, \quad \mathrm{y} \quad \frac{\partial \mathrm{U}}{\partial \mathrm{t}}(\mathrm{x}, 0)=0, \quad 0 \leq \mathrm{x} \leq 1$

Proporciona el estado inicial del objeto a desplazarse en cualquier punto del eje horizontal $\mathbf{x}$ al tiempo $\mathbf{t}=\mathbf{t}_{\text {o }}$ y en para $\mathbf{t}=\mathbf{t}_{1}$ (es decir para $\mathbf{j}=0$ $y j=1$ )

2.1 Para $\mathbf{t}=\mathrm{t}_{\mathrm{o}}=\mathbf{0}(\mathrm{j}=0)$.

2.1.1 los valores de $U$ en $\left(x_{i}, 0\right)$, para $i=1-9$, $\left(U\right.$ en $\left(x_{i}, t_{0}\right)$, para $i=1-9)$, es decir:

$\mathrm{m}$,

$$
\begin{gathered}
\mathrm{U}\left(\mathrm{x}_{1}, 0\right)=\mathrm{U}(0.1,0)=\operatorname{sen}(\pi(0.1))=0.309 \\
0 \\
\mathrm{U}\left(\mathrm{x}_{2}, 0\right)=\mathrm{U}(0.2,0)=\operatorname{sen}(\pi(0.2))= \\
0.5878 \\
\mathrm{U}\left(\mathrm{x}_{3}, 0\right)=\mathrm{U}(0.3,0)=\operatorname{sen}(\pi(0.3))= \\
0.8090 \\
\mathrm{U}\left(\mathrm{x}_{4}, 0\right)=\mathrm{U}(0.4,0)=\operatorname{sen}(\pi(0.4))= \\
0.9511 \\
\mathrm{U}\left(\mathrm{x}_{5}, 0\right)=\mathrm{U}(0.5,0)=\operatorname{sen}(\pi(0.5))= \\
1.0000 \\
\mathrm{U}\left(\mathrm{x}_{6}, 0\right)=\mathrm{U}(0.6,0)=\operatorname{sen}(\pi(0.6))= \\
0.9511 \\
\mathrm{U}\left(\mathrm{x}_{7}, 0\right)=\mathrm{U}(0.7,0)=\operatorname{sen}(\pi(0.7))= \\
0.8090 \\
\mathrm{U}\left(\mathrm{x}_{8}, 0\right)=\mathrm{U}(0.8,0)=\operatorname{sen}(\pi(0.8))= \\
0.5878 \\
\mathrm{U}\left(\mathrm{x}_{9}, 0\right)=\mathrm{U}(0.9,0)=\operatorname{sen}(\pi(0.9))= \\
0.3090
\end{gathered}
$$

2.1.2. Para los valores en $U(0,0)$ y $U(1,0)$; se tiene según las condiciones Inicial es: $U(0,0)=0$ y $U(1,0)=0$.

2.1.3. Por lo tanto los valores de la temperatura para las condiciones Iniciares son:
$\mathrm{U}_{0,0}=0 \quad \mathrm{U}_{1,0}=0.3090$
$\mathrm{U}_{2,0}=0.5878$
$\mathrm{U}_{3,0}=0.8090 \mathrm{U}_{4,0}=0.9511$
$\mathrm{U}_{5,0}=1$
$\mathrm{U}_{6,0}=0.9511 \mathrm{U}_{7,0}=0.8090$
$\mathrm{U}_{8,0}=0.5878$
$\mathrm{U}_{9,0}=0.3090 \mathrm{U}_{10,0}=0$

2.1. Para $\left.\mathrm{t}=\mathrm{t}_{1}=0.01 \quad \mathrm{j}=1\right)$.

2.2.1 Los valores de $U$ en $\left(x_{i}, 0.01\right)$, para $i=1-9$, $\left(U\right.$ en $\left(x_{i}, t_{1}\right)$, para $\mathrm{i}=1-9)$. Para una mejor aproximación, usamos:

$U_{i, 1}=U\left(x_{i}, t_{1}\right) \approx\left(1-\lambda^{2}\right) f\left(x_{i}\right)+\frac{\lambda^{2}}{2} f\left(x_{i+1}\right)+\frac{\lambda^{2}}{2} f\left(x_{i-1}\right)+k g\left(x_{i}\right)$

$$
\mathrm{U}\left(\mathrm{x}_{1}, 0.01\right)=\mathrm{U}(0.1 \text {, }
$$

\begin{tabular}{|c|c|}
\hline $\mathrm{U}_{0,20}=0$ & $\mathrm{U}_{10,20}=0$ \\
\hline$\vdots$ & $\vdots$ \\
\hline $\mathrm{U}_{0,10}=0$ & $\mathrm{U}_{10,10}=0$ \\
\hline $\mathrm{U}_{0,9}=0$ & $\mathrm{U}_{10,9}=0$ \\
\hline $\mathrm{U}_{0,8}=0$ & $\mathrm{U}_{10,8}=0$ \\
\hline $\mathrm{U}_{0,7}=0$ & $\mathrm{U}_{10,7}=0$ \\
\hline $\mathrm{U}_{0,6}=0$ & $\mathrm{U}_{10,6}=0$ \\
\hline $\mathrm{U}_{0,5}=0$ & $\mathrm{U}_{10,5}=0$ \\
\hline $\mathrm{U}_{0,4}=0$ & $\mathrm{U}_{10,4}=0$ \\
\hline $\mathrm{U}_{0,3}=0$ & $\mathrm{U}_{10,3}=0$ \\
\hline $\mathrm{U}_{0,2}=0$ & $\mathrm{U}_{10,2}=0$ \\
\hline $\mathrm{U}_{0,1}=0$ & $\mathrm{U}_{10,1}=0$ \\
\hline $\mathrm{U}_{0,0}=0$ & $\mathrm{U}_{10,0}=0$ \\
\hline
\end{tabular}

$0.01)=\operatorname{sen}(\pi(0.1))=0.3090$

$\mathrm{U}\left(\mathrm{x}_{2}, 0.01\right)=\mathrm{U}(0.2,0.01)=\operatorname{sen}(\pi(0.2))=$ 0.5878

$\mathrm{U}\left(\mathrm{x}_{3}, 0.01\right)=\mathrm{U}(0.3,0.01)=\operatorname{sen}(\pi(0.3))=$ 0.8090

$\mathrm{U}\left(\mathrm{x}_{4}, 0.01\right)=\mathrm{U}(0.4,0.01)=\operatorname{sen}(\pi(0.4))=$ 0.9511

$U\left(x_{5}, 0.01\right)=U(0.5,0.01)=\operatorname{sen}(\pi(0.5))=$ 1.0000

$U\left(x_{6}, 0.01\right)=U(0.6,0.01)=\operatorname{sen}(\pi(0.6))=$ 0.9511

$\mathrm{U}\left(\mathrm{x}_{7}, 0.01\right)=\mathrm{U}(0.7,0.01)=\operatorname{sen}(\pi(0.7))=$ 0.8090

$\mathrm{U}\left(\mathrm{x}_{8}, 0.01\right)=\mathrm{U}(0.8,0.01)=\operatorname{sen}(\pi(0.8))=$ 0.5878

$\mathrm{U}\left(\mathrm{x}_{9}, 0.01\right)=\mathrm{U}(0.9,0.01)=\operatorname{sen}(\pi(0.9))=$ 0.3090

3. Para la condición de frontera son:

Figura 4: resultados de condición de frontera

Fuente: propia 


\section{Usando Matlab}

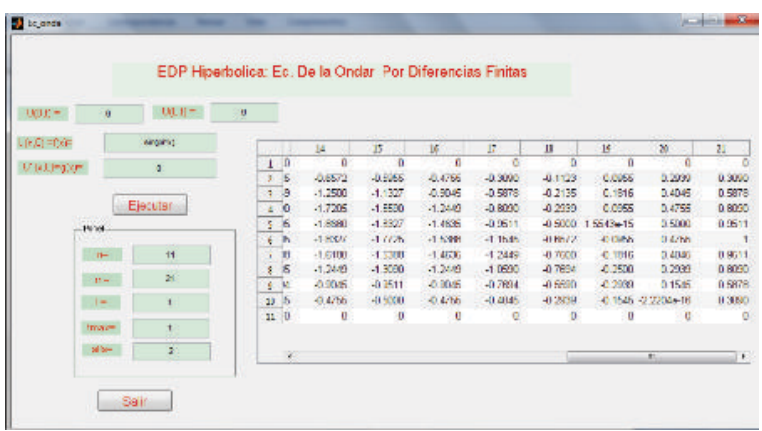

Figura 5: resultado final usando MATLAB

Fuente: Propia

\section{Curvas:}

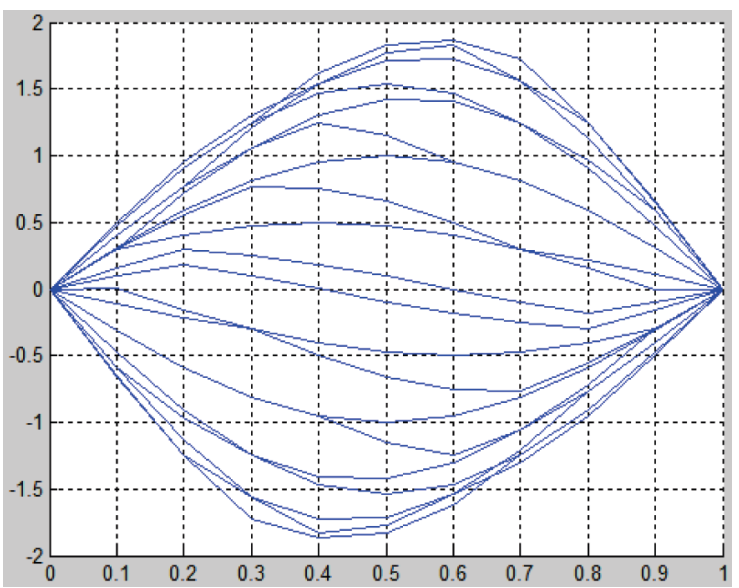

Figura 6: Representación gráficas en $\mathrm{R}^{2}$ de las soluciones.

Fuente: Propia

\section{Superficie}

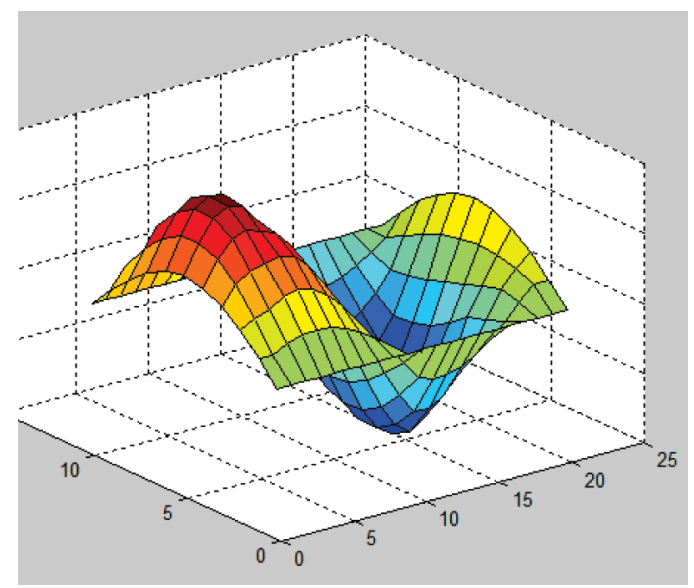

Figura 7: Representación gráficas en $\mathrm{R}$ de las soluciones.

Fuente: Propia
Programa compuatcional hecho en Matlab

function pushbutton1_Callback(hObject, eventdata, handles)

close(Ec onda);

$\%$--- Executes on button press in pushbutton2.

function pushbutton2_Callback(hObject, eventdata, handles)

Uot=inline(get(handles.edit1,'string')); ULt=inline(get(handles.edit9,'string'));

$\mathrm{f}=$ inline(get(handles.edit2,'string')); $g=$ inline(get(handles.edit3,'string')); n=str2double(get(handles.edit4,'string')); $\%$ n=numero de columnas m=str2double(get(handles.edit5,'string')) $; \% n=$ numero de columnas

$\mathrm{L}=$ str2double(get(handles.edit6,'string')); tmax =str2double(get(handles.edit7,'strin $\left.\left.g^{\prime}\right)\right)$;

alfa=str2double(get(handles.edit8,'string '));

$\mathrm{h}=\mathrm{L} /(\mathrm{n}-1)$

$\mathrm{k}=\mathrm{tmax} /(\mathrm{m}-1)$

landa $=$ alfa* $k / h$

$\mathrm{U}=$ zeros $(\mathrm{n}, \mathrm{m})$

for $i=2: n-1$

$\mathrm{U}(\mathrm{i}, 1)=\mathrm{feval}\left(\mathrm{f},(\mathrm{i}-1)^{*} \mathrm{~h}\right)$;

$\mathrm{U}(\mathrm{i}, 2)=(1-\text { landa^})^{\wedge}{ }^{\star}$ feval $(\mathrm{f},(\mathrm{i}-$

$\left.1)^{*} h\right)+((\text { landa^} 2) / 2)^{*}\left(\right.$ feval $\left.\left(f, i^{*} h\right)\right)+f e v a l(f,($ $\left.\mathrm{i}-2)^{\star} \mathrm{h}\right)+\mathrm{k}^{\star} \mathrm{feval}\left(\mathrm{g},(\mathrm{i}-1)^{\star} \mathrm{h}\right)$;

end

for $\mathrm{j}=3: \mathrm{m}$

for $i=2: n-1$

$U(i, j)=2^{*}(1-\text { landa^ } 2)^{*} U(i, j-$

1) $+(\text { landa^} 2)^{*}(U(i+1, j-1)+U(i-1, j-1))-U(i, j-$ 2);

end

end

for $\mathrm{j}=1: \mathrm{m}$

$\mathrm{t}=(\mathrm{j}-1)^{*} \mathrm{k}$;

for $i=1: m$ $\mathrm{x}=(\mathrm{i}-1)^{*} \mathrm{~h}$;

end

end

$\mathrm{U}$ 


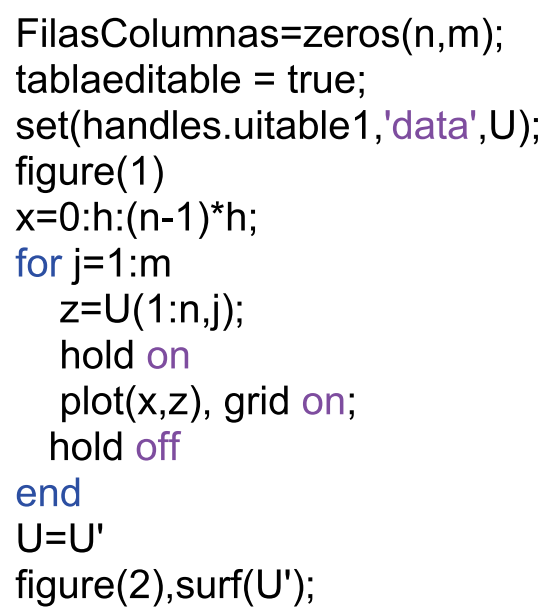

\section{CONCLUSIONES}

Se Aplicó las diferencias finitas para una solución numérica de la ecuación diferencial parcial

Se Aplicó las diferencias finitas para una solución numérica de la ecuación de la onda

\section{REFERENCIAS BLIBLIOGRÁFICAS}

Chapra, Steven y Canales, Raymond, (2007). Métodos Numéricos para Ingenieros; 5ta. Edición, Ed. Mc Graw HILL; México.

Burden R. y Faires D. (2007). Análisis Numérico. México, D.F.: Iberoamérica

Nieves H. Y Domínguez F. (1997). Métodos Numéricos. ( $2^{\circ}$ edic.). México, D.F.: Continental

Curtis G. (1992). Análisis Numérico. México, D.F.: Ediciones Alfa y Omega

CHAPRA S. (2007). Métodos numéricos. (2 edic.). Mexico: Mc Graw Hill

Nieves H. Y Domínguez F. (2007). Métodos Numéricos. (5ta. edic.). México, D.F.: Continental

Infante J. y Rey J. (2007). Métodos Numéricos/Numerical Methods: Teoría, problemas y prácticas con Matlab/Theory, Problems and Matlab Practices. 